\title{
Erratum
}

\section{Erratum to: Unstable Surface Waves in Running Water}

\author{
Vera Mikyoung Hur ${ }^{1}$, Zhiwu Lin ${ }^{2}$ \\ ${ }^{1}$ Department of Mathematics, University of Illinois at Urbana-Champaign, Urbana, IL 61801, USA. \\ E-mail: verahur@math.uiuc.edu \\ 2 School of Mathematics, Georgia Institute of Technology, Atlanta, GA 30332, USA. \\ E-mail: zlin@math.gatech.edu
}

Received: 7 September 2011 / Accepted: 20 September 2011

Published online: 6 February 2013 - @ C Springer-Verlag Berlin Heidelberg 2013

Commun. Math. Phys. 282, 733-796 (2008)

It was pointed out by Michael Renardy that there were a few errors in Sect. 4 and Sect. 6 of [1]. First, in [1, Prop. 4.4] it was claimed that for a class of shear flows the wave speed of a neutral limiting mode must be the inflection value. As indicated in [2] however two other possible values of the wave speed must be added, namely, an extremum value and the value at the bottom of the shear flow. We give the correct statement. Accordingly, we make changes to the statements of several assertions in Sect. 4 and Sect. 6 of [1]. Second, the proof of [1, Lem. 4.11] contains an error. We give a correct proof, which works for a more general class of shear flows. We note that these changes do not affect the main conclusions of [1]; see Remark 4.

Throughout, we use the notation in [1] and study the Rayleigh equation

$$
(U-c)\left(\phi^{\prime \prime}-\alpha^{2} \phi\right)-U^{\prime \prime} \phi=0 \text { in }(0, h)
$$

with the boundary conditions

$$
\left\{\begin{array}{l}
(U(h)-c)^{2} \phi^{\prime}(h)=\left(g+U^{\prime}(h)(U(h)-c)\right) \phi(h), \\
\phi(0)=0 .
\end{array}\right.
$$

The main correction is to change [1, Prop. 4.4] to the following.

Proposition 4.4. For $U \in \mathcal{K}^{+}$, a neutral limiting mode $\left(\phi_{s}, \alpha_{s}, c_{s}\right)$ must be one of the following three cases:

(1) $c_{s}=U_{s}$ (inflection value) and $\phi_{s}$ solves (1)-(2) with $c=U_{s}$;

(2) $c_{s}=U(0)$ and $\phi_{s}>0$ on $(0, h)$ solves (1)-(2) with $c=U(0)$; 
(3) $c_{s}=U_{c}$, an extremum value of $U$; if $y_{c} \in(0, h)$ is the largest such that $U\left(y_{c}\right)=U_{c}$, then $\phi_{s}(y)=0$ for $y \in\left[0, y_{c}\right]$ and $\phi_{s}(y)>0$ for $y \in\left(y_{c}, h\right)$ solves $(1)$ with the boundary conditions

$$
\left\{\begin{array}{l}
\left(U(h)-c_{s}\right)^{2} \phi_{s}^{\prime}(h)=\left(g+U^{\prime}(h)\left(U(h)-c_{s}\right)\right) \phi_{s}(h), \\
\phi_{s}\left(y_{c}\right)=0, \phi_{s}^{\prime}\left(y_{c}\right)=0 .
\end{array}\right.
$$

The case (2) can occur only when $U(y)>U(0)$ or $U(y)<U(0)$ for $y \in(0, h]$.

The proof of that $c_{s}=U_{s}, U(0)$ or $U_{c}$ is sketched in [2], completing the arguments in [1]. We will show properties of $\phi_{s}$ in the cases of (2) and (3).

Suppose that $c_{s} \neq U_{s}$ or $U(0)$. Let $\tilde{y} \in(0, h]$ be the largest such that $U(\tilde{y})=c_{s}$. It is shown in [1] that $\phi_{s}$ is not identically zero and $\phi_{s}(y)=0$ for $y \in[0, \tilde{y}]$. We claim that $\phi_{s}$ vanishes nowhere on $(\tilde{y}, h)$. Suppose otherwise that $\phi_{s}(\bar{y})=0$ for some $\bar{y} \in(\tilde{y}, h)$. Then, $\phi_{s} \equiv 0$ on $[\tilde{y}, \bar{y}]$ by [1, Lem. 4.7]. Since the ordinary differential equation (1) is regular in $[\bar{y}, h]$, furthermore, $\phi_{s} \equiv 0$ on $[\bar{y}, h]$. A contradiction therefore proves the claim, and we may assume that $\phi_{s}>0$ on $(\tilde{y}, h)$. If $U^{\prime}(\tilde{y}) \neq 0$, then from $\phi_{s}(\tilde{y})=\phi_{s}^{\prime}(\tilde{y})=0$ and from the $H^{2}$-bound in [1, Lem. 4.6] of $\phi_{s}$, we deduce that $\phi_{s} \equiv 0$ in a neighborhood of $\tilde{y}$ by the property of solutions of (2) near $\tilde{y}$. Thus, $c_{s}$ must be an extremum and $\phi_{s}$ has the stated properties. If $c_{s}=U(0)$ which is not an extremum value then $U-c_{s} \neq 0$ on $(0, h)$. Otherwise, $\phi_{s} \equiv 0$ on $(0, h)$ by the above argument. If $c_{s}=U(0)$ and $U(y)=U(0)$ only at $y=0$, then $\phi_{s}$ cannot vanish on $(0, h]$ and hence we may assume that $\phi_{s}>0$ on $(0, h]$.

As pointed out in [2], the mistake in [1] was to conclude that $\phi_{s}=0$ on $[0, \tilde{y}]$ would imply $\phi_{s}=0$ on $[0, h]$ assuming $c_{s} \neq U_{s}$ only; the cases $c_{s}=U(0)$ and an extremum value were neglected.

Remark 1. As shown in [2] by means of examples and numerical computations, all three cases of neutral limiting modes $c=U(0)$, an inflection value and an extremum value can occur. In the case of $c_{s}=U(0)$ or an extremum value, in view of Proposition 4.4 the wave number $\alpha_{s}$ is unique.

We must change the statement of [1, Thm. 4.2] accordingly. Let $\alpha_{0}$ be the wave number of the neutral limiting mode with $c=U(0)$ and $\alpha_{1}, \ldots, \alpha_{k}$ be waves numbers of neutral limiting modes with $c$ an extremum value of $U$.

Theorem 4.2. For $U \in \mathcal{K}^{+}$, suppose that $-\alpha_{\max }^{2}<0$ is the lowest eigenvalue of the ordinary differential operator $-\frac{d^{2}}{d y^{2}}-K$ on the interval $(0, h)$ with the boundary conditions (4.5)-(4.6) in [1]. Let

$$
\bar{\alpha}= \begin{cases}0 & \text { if } \min \left\{\alpha_{0}, \ldots, \alpha_{k}\right\} \geq \alpha_{\max }, \\ \max \left\{\alpha_{i} \mid \alpha_{i}<\alpha_{\max }\right\} & \text { if } \min \left\{\alpha_{0}, \ldots, \alpha_{k}\right\}<\alpha_{\max }\end{cases}
$$

Then, to each $\alpha \in\left(\bar{\alpha}, \alpha_{\max }\right)$ there corresponds an unstable solution triple $(\phi, \alpha, c)$ (with $\operatorname{Im} c>0)$ of (1)-(2).

The proof is a minor modification of that in [1]. It is shown in [1] that for $U \in$ $\mathcal{K}^{+}$, unstable modes bifurcate near a neutral mode with $c=U_{s}$ (inflection value) and $\alpha=\alpha_{\max }$ for $\alpha$ slightly less than $\alpha_{\max }$. The unstable mode then continues for smaller $\alpha$. Since the continuation can only stop at a neutral limiting mode with $c=U(0)$ or an 
extremum value, there is an unstable mode for any $\alpha \in\left(\bar{\alpha}, \alpha_{\max }\right)$. Of course, $\left(\bar{\alpha}, \alpha_{\max }\right)$ may not be the sharp interval for instability. To get a completed picture, one needs to study the local bifurcation of unstable modes near neutral modes with $c=U(0)$ or an extremum value.

In view of [1, Lem. 4.11] and the corrected Theorem 4.2, we make changes to the statement of [1, Cor. 4.12].

Corollary 4.12. A monotone shear flow $U$ with exactly one inflection point $y_{s}$ and $-U^{\prime \prime} /\left(U-U\left(y_{s}\right)\right)>0$ is unstable in the free surface setting, for wave numbers in the interval $\left(\bar{\alpha}, \alpha_{\max }\right)$, where

$$
\bar{\alpha}= \begin{cases}0 & \text { if } \alpha_{0} \geq \alpha_{\max } \\ \alpha_{0} & \text { if } \alpha_{0}<\alpha_{\max }\end{cases}
$$

and $\alpha_{0}, \alpha_{\max }>0$ are as defined in Theorem 4.2.

In Sect. 6 of [1], we made the same mistake as in [1, Prop. 4.4] in characterizing neutral limiting modes for the class $\mathcal{F}$. Lemma 6.2 and Proposition 6.6 of [1] must be replaced by the following.

Lemma 6.2. For $U \in \mathcal{F}$, we have the same characterization of neutral limiting modes as in Proposition 4.4.

For the proof, we first derive an $\mathrm{H}^{2}$-bound for the sequence of unstable modes approaching a neutral limiting mode. Then, the same arguments as in the proof of Proposition 4.4 apply. The $H^{2}$-bound can be obtained by a slight adjustment of the proof of [1, Lem. 4.6].

Changes are required to several assertions in $[1$, Sect. 6]. The conclusions of Lemma 6.3 and Theorem 6.4 in [1] must be dropped. In fact, it was claimed in [1, Thm. 6.4] that a shear flow without inflection is stable. But, it was shown in [2] by numerical computations that $U(y)=1-y^{2}$ for $y \in(-1,1)$ has unstable modes for wave number in $\left(\alpha_{\min },+\infty\right)$, where $\alpha_{\min }$ is the wave number of the neutral mode with $c$ the extremum value 1 . The description in [1, Thm. 6.7] of the interval of unstable wave numbers must include the cases of the neutral modes with $c=U(0)$ and extremum values in addition to the case with $c$ inflection values.

Another corrigendum is in the proof of [1, Lem. 4.11] due to the incorrect use of the Sturm-Liouville comparison theorem. We fix it in the following.

Proof of Lemma 4.11 in [1]. Let $K=-U^{\prime \prime} /\left(U-U_{s}\right)$ and $\mu_{0}$ be the lowest eigenvalue of $-\frac{d^{2}}{d y^{2}}-K$ in $(0, h)$ with the Dirichlet boundary conditions at $y=0$ and $y=h$. We consider three cases.

Case 1. $\mu_{0}<0$. By the argument in [1, Rem. 4.10], the lowest eigenvalue of $-\frac{d^{2}}{d y^{2}}-K$ with the boundary conditions (2) for $c=U_{s}$ is negative, which we denote by $-\alpha^{2}$. Then, a solution to (1)-(2) is found for $c=U_{s}$ and $\alpha$.

Case 2. $\mu_{0}>0$. We modify the arguments in the proof of [1, Lem. 4.11]. Let $\phi_{\alpha}$ be the solution of the Rayleigh equation 


$$
\phi_{\alpha}^{\prime \prime}+\left(K-\alpha^{2}\right) \phi_{\alpha}=0 \text { in }(0, h)
$$

with $\phi_{\alpha}(0)=0$ and $\phi_{\alpha}^{\prime}(0)=1$. We claim that

$$
\phi_{\alpha}>0 \text { on }(0, h] \text { for any } \alpha \geq 0 .
$$

Suppose on the contrary that there exists $\alpha_{0} \geq 0$ such that $\phi_{\alpha_{0}}$ has a zero on $(0, h]$. Let $y_{0} \in(0, h]$ be such that $\phi_{\alpha_{0}}(y)>0$ for $y \in\left(0, y_{0}\right)$ and $\phi_{\alpha_{0}}\left(y_{0}\right)=0$. Thus, $-\alpha_{0}^{2}$ is the lowest eigenvalue of $-\frac{d^{2}}{d y^{2}}-K$ on $\left(0, y_{0}\right)$ with the Dirichlet boundary conditions $\phi(0)=0=\phi\left(y_{0}\right)$. Since the lowest eigenvalue of $-\frac{d^{2}}{d y^{2}}-K$ in $(0, a)$ with the zero Dirichlet conditions is decreasing in $a \in(0, h]$, it follows that $\mu_{0} \leq-\alpha_{0}^{2} \leq 0$. A contradiction then proves (3). The rest of the proof is the same as that in [1].

Case 3. $\mu_{0}=0$. The same argument as in Case 2 shows that $\phi_{\alpha}>0$ on $(0, h]$ for any $\alpha>0$. Note that $\phi_{0}(h)=0$. Since $\phi_{0}$ is the zero eigenfunction of $-\frac{d^{2}}{d y^{2}}-K$ on $(0, h)$ with the zero boundary conditions, $\lim _{\alpha \rightarrow 0+} \phi_{\alpha}(h)=0$. Hence,

$$
\begin{aligned}
f(\alpha) & =\frac{U(0)-U_{s}}{\left(U(h)-U_{s}\right) \phi_{\alpha}(h)}+\frac{\alpha^{2}}{\left(U(h)-U_{s}\right) \phi_{\alpha}(h)} \int_{0}^{h}\left(U-U_{s}\right) \phi_{\alpha} d y-\frac{g}{\left(U_{s}-U(h)\right)^{2}} \\
& =\frac{1}{\phi_{\alpha}(h)}\left[\frac{U(0)-U_{s}}{U(h)-U_{s}}+\frac{\alpha^{2}}{\left(U(h)-U_{s}\right)} \int_{0}^{h}\left(U-U_{s}\right) \phi_{\alpha} d y\right]-\frac{g}{\left(U_{s}-U(h)\right)^{2}}
\end{aligned}
$$

defined in [1, p. 768] satisfies that $\lim _{\alpha \rightarrow 0+} f(\alpha)=-\infty$. The rest of the proof again follows by that in [1].

Remark 2. Indeed, [1, Lem. 4.11] holds true for any non-monotone $U$ satisfying that $K$ is bounded and $U_{s}$ is between $U(0)$ and $U(h)$.

Remark 3. If $U(0)$ is the strict absolute minimum (or maximum) and $c_{s}=U(0)$ then

$$
-\alpha_{s}^{2}=\inf _{\substack{\phi \in H^{1}(0, h) \\ \phi(0)=0}} \frac{\int_{0}^{h}\left(\left|\phi^{\prime}(y)\right|^{2}+\frac{U^{\prime \prime}(y)}{U(y)-U(0)}|\phi(y)|^{2}\right) d y-\left(\frac{g}{(U(h)-U(0))^{2}}+\frac{U^{\prime}(h)}{U(h)-U(0)}\right)|\phi(h)|^{2}}{\int_{0}^{h}|\phi(y)|^{2} d y} .
$$

Choosing the test function $\phi=U-U(0)$, we have

$$
-\alpha_{s}^{2} \leq-\frac{g}{\int_{0}^{h}(U-U(0))^{2} d y}<0 .
$$

Thus, there exists a unique neutral limiting mode with $c=U(0)$ for any $g>0$. If $g=0$ then, obviously, $\alpha_{s}=0$ and $\phi_{s}=U-U(0)$. For $g$ small, $\alpha_{s}=O(\sqrt{g})$ and $\alpha_{s} \rightarrow+\infty$ as $g \rightarrow \infty$. Therefore, by Corollary 4.12 , we obtain instability of monotone $U$ with $\frac{U^{\prime \prime}}{U-U\left(y_{s}\right)}<0$, for wave lengths as long as $O(1 / \sqrt{g})$. We must note that although a neutral mode with $c=U(0)$ always exists for any $g$ when $U(0)$ is the strict minimum (or maximum) it may not be contiguous to unstable modes. In fact, in [3, Thm. 2(a)] was found an example of a monotone flow $U$ satisfying $U^{\prime \prime}<0$ and $U^{\prime}(h) \geq 0$, which is stable for any wave number and $g>0$. 
Remark 4. The main results in [1] are not affected by the above changes. An important example used in [1] is

$$
U(y)=A \sin (\beta(y-h / 2)), \quad y \in[0, h],
$$

for any $h, \beta>0$ satisfying $h \beta \leq \pi$ and for an arbitrary $A$. By the corrected Corollary 4.2 , such shear flow is unstable for wave numbers in the interval $\left(\bar{\alpha}, \alpha_{\max }\right)$, instead of $\left(0, \alpha_{\max }\right)$ as claimed in [1]. As shown in [1], there bifurcate small-amplitude periodic water waves with the shear background (4) for any wave number $\alpha$. These periodic waves are unstable when $\alpha \in\left(\bar{\alpha}, \alpha_{\max }\right)$, supporting two conclusions in [1]: first, the bifurcation of periodic water waves is unrelated to stability of the background flow; second, an arbitrarily small vorticity to the irrotational flow of an arbitrary depth may induce instability of water waves. We refer the reader to Remarks 4.14 and 5.2 in [1] for the detail.

We also want to take this opportunity to correct a few typos: on p. 756, Eq. (4.8a), the + before $g_{r}\left(U_{s}\right)$ should be - ; on p. 757, line -2 , the + before $K(y)$ should be - .

\section{References}

1. Hur, V., Lin, Z.: Unstable surface waves in running water. Commun. Math. Phys. 282, 733-796 (2008)

2. Renardy, M., Renardy, Y.: On the stability of inviscid parallel shear flows with a free surface. J. Math. Fluid Mech., to appear, doi:10.1007/s00021-012-0097-4, 2012

3. Yih, C.-S.: Surface waves in flowing water. J. Fluid. Mech. 51, 209-220 (1972)

Communicated by P. Constantin 